\title{
Teaching Ethics in Mathematics
}

Maurice Chiodo (King's College, Cambridge, UK) and Piers Bursill-Hall (The University of Cambridge, UK)

In the previous article in this issue of the Newsletter we addressed why mathematicians should consider the ethics of what they do. Here we outline, based on our experiences, three key elements for teaching Ethics in Mathematics (EiM): (1) a lecture series on ethical issues in mathematics; (2) exercises with an ethical component in problem sheets of other maths courses; and (3) a supportive environment so students perceive value in this teaching.

\section{Why is this an issue now?}

While some may argue that mathematicians will inevitably develop ethical skills when they begin to work in industry, we would like to suggest (see [2]) that the mathematical community actively encourages mathematical professionals to either regard mathematics as beyond ethics (Platonism), or that social and ethical consequences are just "not a mathematician's problem" (exceptionalism). Compare this to law, medicine, physical sciences; etc., which all teach profession-specific ethics. We suggest that, because mathematicians engage in sophisticated technical work which lies well above the level of public scrutiny, they should be actively trained to deepen the awareness of their social and ethical responsibilities (see [1]). But why hasn't it been done before?

Firstly, until the middle of the 20th century most people studying mathematics at post-secondary institutions in the West also received a robust training in philosophy, and thus were equipped with enough philosophical and ethical literacy to deal with professional, ethical questions. The second reason is more profound and lies in the newfound immediacy of the work of mathematicians. Until recently there was a genuine separation between people who did abstract mathematics (mathematicians), and those who applied such work in the physical world (physicists and engineers). There was a discernible professional and temporal gap between those who produced new theorems, and those applying them decades, even centuries, later. This reduced the appearance of ethical responsibility of mathematicians and gave everyone more time to consider the ethical issues. However, the digital revolution has reduced this gap.

The amount of pure mathematics used in finance, surveillance, big data and decision-making systems is vast and growing rapidly. Mathematics has not only become their foundation, but it is being 'brought to market' and has a potential social impact within months or even weeks. The power of new mathematics in ethically-laden industries means the professional and temporal gap between its creation and its application has reduced so much that the ethical consequences of mathematical work cannot be obscured or blamed on someone else. For the first time ever, mathematicians are uniquely responsible for the immediate social consequences of their work.

\section{Constructing a course in EiM}

Teaching Ethics in Mathematics (EiM) turns out to be non-trivial. Since no one has done it before, there is no body of precedent, resources, textbooks or lecture notes from others to build on. ${ }^{1}$ Introducing it as an undergraduate course is necessarily a complex process: its ideas are new to your university, it is unlike theorem-based courses, and sometimes it is hard to argue that EiM should supplant any given traditional maths course, as teaching time and resources are already limited. What follows is only indicative, based on our experiences and conversations with colleagues around the UK and elsewhere in Europe. There is no unique or established way to teach EiM, and you will need to tailor the discussions here to your situation. Treat what follows as suggestions, not rules. We have introduced EiM in Cambridge as an informal, non-examinable course (of which there are already well-attended examples in our Faculty). Students were used to this format, but it might not be the right model for your institution; seminars, compulsory modules or project courses might work better.

It may be best - and easiest - to introduce EiM in a slow, evolutionary way, starting with 1-2 lunchtime seminars before developing it further. It helps your colleagues to get used to the idea, and if it proves to be popular, this may provide its own rationale for the course to be accepted into the curriculum. You will need to consider your audience carefully. Are your students studying mostly maths, or maths with physics or computer science? Different allied disciplines will suggest different case studies.

Teaching EiM is quite different from ethics courses in other disciplines. They do not follow the same exceptionalism and are already aware of the existence of ethical issues. Unfortunately, as mathematicians, we do not have this luxury. Indeed, you probably need to assume that most of your audience does not initially and intuitively accept the premise that there are ethical issues in mathematics. Thus, we strongly suggest not starting an EiM course with generic philosophical discussions on ethics because you can lose your audience as a result. We have found that most of our students are generally not receptive to the conceptual structures, language and approach of 'real' philosophy; what philosophers talk about is not always easily translated to an undergraduate mathematician. Hence, we strongly recommend resisting the temptation to ask a philosopher to teach this. Students need to see profession-specific ethical issues and discussions in a familiar language. Of course, engage with philosophers and ethicists to help design your course, and go to other disciplines (law, social sciences, engineering, etc.) to

\footnotetext{
1 We have constructed a website ethics.maths.cam.ac.uk hosting resources for anyone who wants to construct an EiM course.
} 
get advice and insight. However, we believe that such a course needs to be delivered by mathematicians even if they are not professionally trained ethicists, just like linear algebra lecturers need not be experts in algebra. You probably know more about it than your students, and you speak the same professional language.

A natural structure for such a course would be to split it into two sections: "There exist some ethical issues in mathematics", and then "For all mathematics that we do, there are ethical issues". It may seem pedestrian, but an array of case studies prove their existence. To get students to appreciate it you only need to present explicit and varied examples of work that mathematicians have done which have raised ethical issues. Your audience must reach the point where they accept that there are indeed ethical issues in all branches of mathematics. Giving just one example may lead them to think that it was a one-off. You can find a long list of case studies at ethics.maths. cam.ac.uk/cases; such mathematicians were probably not deliberately acting maliciously, but instead overlooked ethical consequences. It is important to emphasise this; teaching EiM should not be a platform for criticising others, or you risk putting your students on the defensive.

Now you are ready to move to the second stage: "For all mathematics that we do, there are ethical issues". Such generalisation is harder to accept. Students may think that "there are places $\mathrm{X}, \mathrm{Y}, \mathrm{Z}$ where mathematicians might do unethical things, so if I just avoid those, I'm safe." We have had this reaction from our students regularly. You need to dispel this and show them that there is nowhere to hide, not even in academia. Obviously for all statements cannot be proved by example but require more profound arguments. These can build on the lack of sufficient 'external' control mechanisms (weak regulation) and on the fact that mathematicians are trained and encouraged to strip away non-mathematical aspects of problems (which inevitably leads to issues). It can also include more social aspects such as there are people who will deliberately set out to exploit others and their labour, playing on their unwillingness to think about ethical consequences. Your students are about to enter an industrial economy which is set up and organised to work in ways that can obscure the ethical context and can enable moral disengagement.

This is your 'proof for ethics'. No matter how supposedly pure your (mathematical) work is, someone is inevitably paying you to do this work for their interests. When working mathematicians ask: Who is paying me? Why are they paying me? How will they use my work? How will they use me? ? $^{2}$...then an ethical self-examination has begun.

\section{The course we give}

Our course involves 20 contact hours, divided between lectures, interactive exercises and often lively and challenging discussions. We recommend you encourage interaction so students explore and digest these new ideas.

\footnotetext{
2 As the now-resigned director of the MIT Media Lab, Joi Ito, recently found out; tinyurl.com/yyowldy9.
}

Discussions are useful and necessary to develop an ethical understanding. We cover eight topics. The first half illustrate the existence of ethics in mathematics before we move on to argue for its universality, where we have found ourselves appealing to other disciplines (psychology, law, social science) to understand the inner workings of mathematical community and its interactions with the world. On many occasions these extra-mathematical observations proved to be the most interesting and persuasive for our audience. Descriptions, and a recording, of our lectures, are available at ethics.maths.cam.ac.uk/ course/lectures.

In our lectures we cover the following.

\section{(1) Introduction to EiM.}

All mathematics is done in a social context. It sits at the heart of technological advancement and industrial progress. Understanding that it can be used for good, and ill, is the first step to ethical awareness.

(2) Mathematics and modelling.

Mathematical models are necessary to understand the world. We draw on examples from fields such as finance to teach the process of modelling and its limitations. The global financial crisis demonstrates that poorly understanding models can have devastating consequences.

(3) Cryptography, surveillance and privacy.

Mathematicians can enable the infringement of privacy by breaking strong encryption, collecting troves of personal data or through carelessness.

(4) Fairness and impartiality in algorithms and AI.

We talk about the ethics behind automated decisionmaking systems and related problems of fairness and impartiality by drawing on examples from predictive policing, prison sentencing, targeted advertising and mathematical fairness measures.

(5) Regulation, accountability and the law.

Industrial mathematics is very close to its social impact (e.g., credit scoring via machine learning) and hence mathematicians need to reconsider their responsibility, understand laws and regulations, and learn to selfregulate when lawmakers are behind the times.

(6) Understanding the behaviour of the mathematical community.

All fields, including mathematics, have a sense of community, conventions and values. Abstraction and the art of mathematical thinking may not necessarily lead to ethical solutions to industrial or social problems.

(7) Psychology 101 - how to survive as a mathematician at work.

Mathematicians encounter other issues, conflicts and dangers arising in the workplace. Their focused and dedicated nature means they may overlook instances of exploitation and manipulation of them and their work. Students must learn to identify these and to protect themselves.

(8) A look into the future, what are the next steps?

Being aware of the ethical issues is not the last step to take. We talk about ways to engage in moral behaviour by talking to colleagues, getting involved with 
decision-making processes and by identifying and calling out unethical and harmful mathematics.

\section{Be interactive!}

We found it extremely fruitful to engage students in interactive demonstrations to show that, even though they are very logical in their thinking, and the problems they work on are well-defined with 'exact' solutions, they are still people with vices, shortcomings and weaknesses. When mathematicians do maths, they do not suddenly become perfect Platonic logical machines. It is essential to dispel the myth that "we're not people, we're mathematicians". For example, you can ask the audience to break up into groups, each producing an impartial plagiarism testing algorithm. Get them to present it to the class, and then proceed to pick apart all the value-judgements presented. If you are lucky, a few students will notice that there is no impartial plagiarism tester! The literature on the psychology of groups is full of valuable (and entertaining) tests and exercises to show how easily one can yield to unspoken social pressures.

Another activity is the 'oil pipe problem' [3, p. 124]. Start by drawing a an oil rig in the ocean and a refinery on a straight shoreline, giving the cost of piping under water and on land. Then ask your students to discuss and compute the optimal pipe path from the rig to the refinery. They may treat it as a first-year calculus problem at which point you should ask what other information might be relevant; are there coral reefs or protected habitats in the vicinity? It teaches students to include soft constraints alongside time and money. Our students quickly became engaged in lively discussions in these examples.

\section{Teaching EiM: Politics or not?}

Will you try to explain what the 'right' ethical conclusions are, expound on moral frameworks, or restrict yourself to only raising ethical awareness without offering answers or solutions? We regularly have students ask us, unsurprisingly, for the 'right answer' or the 'axioms and algorithms of ethics'. While we tried to avoid drawing ethical conclusions, this desire comes up regularly. We strongly suggest aiming to avoid ethical conclusions, and instead getting students to face the difficult job of coming to their own conclusions for their own reasons. By making it political, an anti/pro-capitalist rant, or a mission for social justice, you risk alienating students and colleagues. Many are simply not interested in a political agenda, but do care about not harming people.

Some mathematicians realise that maths has ethical consequences; others do not particularly care whether they cause harm. But most just lack well-developed ethical awareness. They may want to do maths, have fun in the process, and earn a living without causing harm to others; you can thus raise their ethical consciousness, as well as change how they view their work. You do not have to teach them political conclusions; this isn't part of mathematics, but part of the ordinary political discourse citizens have about their political world.

One reason mathematicians shy away from ethical discussions is that mathematics seeks timeless, absolute truths. The apparent perfection of mathematical truth can be its primary attraction. But ethics doesn't have the same binary clarity or timelessness. Different people may come to different conclusions or hold different moral values which are all reasonable, and mathematicians facing profession-specific ethical challenges have no universallyagreed ethical framework to use, because there isn't one. Unsurprisingly, suggesting that mathematicians need to be aware of ethical issues sometimes gets the response that ethics is imperfect and a matter of opinion, and moreover "Whose ethics?" which we would answer with "Yours!" We do not suggest that teaching EiM should give all the answers to ethical problems, but we do suggest that it is our duty to educate our students about it. The hard work of solving the questions remains and is an individual's social responsibility. The political debate that follows is part of what informed citizens frequently do.

\section{Resources, exercise sheets and assessment}

Setting assessment will depend on your course and department. If you do (we didn't), we would suggest setting essay(s) with an emphasis on analysis, reasoning, identification and exploration of ethical issues and (mathematical) sources. Judge contextualisation and line of reasoning, rather than the final conclusion. You can even ask students to present several solutions or options to a particular scenario (hypothetical, or drawing from real life).

There is something more important than assessment. Mathematics is not a spectator sport; every day, maths students go home after lectures and spend many hours on traditional exercise sheet questions. We all know that the value of doing this is to understand the mathematics at a deeper level by 'doing it for yourself'. In the same vein, students need to 'go home and practice' thinking about the ethical issues that can arise when 'doing' mathematics. What we propose is to give students mathematical exercises with real mathematical content, which also have an ethical component. One could assemble a collection of such questions into a set of dedicated sheets, and one might even make this the system of assessment of an EiM course. However, this still compartmentalises the learning process. So let us make a modest proposal: Beyond exercises for an EiM course, we believe it would be more effective if, when doing mathematical exercises in other courses, students encountered questions that require ethical considerations. This could help normalise ethical awareness in everyday mathematics. Its impact could be as large, or larger, than a standalone EiM course. While this is a different order of ethical engagement on the part of the department, it requires minimal effort on the part of your colleagues. If some of the exercise sheets in some of the courses contained a problem or two with an ethical flavour, this might serve to painlessly normalise the ethical engagement and awareness for many students. For first and second-year courses, we have prepared such a collection of questions, which can be found at ethics. maths.cam.ac.uk/course.

Students need to train their ethical reasoning just like they train mathematical reasoning via exercises. This proposition has the benefit that it requires no alteration 
to the core lecture content, beyond simply highlighting in lectures that some example sheet questions are designed to train not only technical and abstract understanding but also the interpretation of mathematics. However, whatever the mechanism your institution uses to give feedback on exercise sheets, you would need to instruct your teaching assistants about these questions. Don't expect them to instantly understand it; they are, after all, mathematicians who probably haven't had much training in ethical awareness. Providing written explanations helps. If example classes are predominantly led by graduate students, then as well as attending your EiM lectures, they can get involved with these EiM questions through teaching them.

\section{Faculty support}

Faculty support is critical to setting up an EiM course, but it can be hard to get. You do not necessarily need your colleagues' time or energy, you just need them to acquiesce to an experiment in EiM, even though it isn't about theorems or applications. In academia where resources are stretched so thinly that we struggle to teach all the mathematics we would like to, you will need to give good arguments to allocate resources to training in ethics. If we are trying to produce the best mathematicians possible and not just maximise the number of theorems taught, we have a duty to teach our students how to use this power and their mathematics responsibly. Otherwise, why are we teaching it to them at all?

Dismissive colleagues can damage the effectiveness of teaching EiM. Phrases such as "Why waste your time going to EiM lectures?", or even more subtle assertions ("Oh, don't worry about question 4; it's one of those ethics questions.") are damaging as they're quickly picked up by students, and it is essential to get departmental leadership on board to encourage colleagues to avoid (directly or indirectly) undermining the credibility of this teaching.

The objections can be orthogonal. One person might say "There is no EiM, so no need to teach it" and another might say "It is obvious that there is EiM, so no need to teach it". However, the most significant objection is an entirely reasonable argument: "We're a maths department, why are we teaching ethics? It's not precise; it's a matter of opinion". As we have repeated ad nauseum, other fields teach profession-specific ethics within their university training. Medical ethics is not medicine, but it makes doctors better doctors. Ethics is a matter of opinion, but that does not mean it cannot be addressed. Mathematicians deal with matters of opinion all the time. We discuss the beauty of mathematics, the elegance of proofs, letters of reference, partial marks on exams, and promotions. When refereeing papers, we fill our reports with value-judgements and opinions beyond mathematical accuracy. When every other profession faces ethical issues and trains professionals to deal with these issues, how can we exclude ourselves from it?

\section{Concluding remarks}

To be eligible for funding for a Centre for Doctoral Training (CDT) from EPSRC, applicants must demonstrate the provision of appropriate training in ethics for all doctoral students. An EiM course would give a convinc- ing response to any such application, demonstrating that the applicants and department genuinely care about ethics and take it seriously. Referees will likely give more weight to an established EiM course than a simple statement of intent to teach ethics, or a reference to an external provider of such 'Responsible Research and Innovation' training (with no specific focus on mathematics).

We have had students from our EiM course tell us they had spoken to large tech companies who were extremely impressed that mathematicians were learning about ethics. It is a highly desirable skill, and as part of your teaching, you may consider providing students with a 'letter of participation'. This may not seem like much, but to employers, a mathematician with any ethical training can be a real asset in today's data-driven economy.

Recently a major UK broadsheet published an editorial arguing that mathematicians need to consider ethics [4]. And the 2019 Royal Institution Christmas lectures, to be delivered by Hannah Fry, will essentially focus on 'ethical issues in mathematics'. If the editors of a newspaper, and the general public, are aware of these issues and of the social responsibilities of mathematicians, surely the time has come to start teaching it to our students.

\section{Acknowledgements}

We wish to thank Dennis Müller for his valuable discussions on this article, and Toby Clifton for his careful editing and many contributions to our work. We also wish to thank Iain Moffatt, as editor of the LMS Newsletter, for his thoughtful contributions on revisions of this article.

This article first appeared in the Newsletter of the London Mathematical Society, Issue 485, November 2019.

\section{References}

1 M. Chiodo, R. Vyas, The role of ethics in a mathematical education, Ethics in Mathematics Discussion Papers, 2018/1 (2018).

2 M. Chiodo, T. Clifton, The Importance of Ethics in Mathematics, EMS Newsletter, December 2019.

3 B. Schulman, Is There Enough Poison Gas to Kill the City? The Teaching of Ethics in Mathematics Classes, College Math. J. 33 (2002) 118-125.

4 The Guardian view on ethics for mathematicians: an essential addition, tinyurl.com/y2pj9tbq, theguardian.com, August 18th 2019.

5 M. Chiodo, P. Bursill-Hall, Four levels of ethical engagement. Ethics in Mathematics Discussion Papers, 2018/1 (2018).

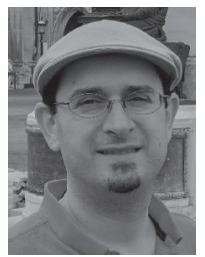

Maurice Chiodo [mcc56@cam.ac.uk] is a Fellow and Teaching Officer at King's College, Cambridge. He is the lead investigator of the Cambridge University Ethics in Mathematics Project (ethics.maths.cam. ac.uk), developing a programme to teach mathematicians about the ethical implications of their work.

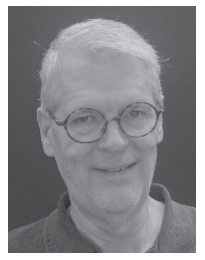

Piers Bursill-Hall [piers@maths.cam.ac.uk] has taught history of maths and science in the mathematics faculty in Cambridge for over 40 years. He has worked extensively on mathematical culture and the status of mathematics in past communities. 